JOURNAL OF

FUNCTION SPACES AND APPLICATIONS

Volume 8, Number 3 (2010), 287-299
(C) 2010, Scientific Horizon http://www.jfsa.net

\title{
Function spaces on the Koch curve
}

\author{
Maryia Kabanava \\ (Communicated by Hans Triebel)
}

2000 Mathematics Subject Classification. 42B35, 42C40, 28A80.

Keywords and phrases. Periodic Besov spaces; trace spaces; self-similar set; Daubechies wavelets.

Abstract. We consider two types of Besov spaces on the Koch curve, defined by traces and with the help of the snowflaked transform. We compare these spaces and give their characterization in terms of Daubechies wavelets.

\section{Introduction}

Let $\Gamma$ be the Koch curve in $\mathbb{R}^{2}$. It is an example of a $d$-set with $d=\frac{\log 4}{\log 3}$. There are two possibilities to introduce Besov spaces on $\Gamma$. The first one is to define Besov spaces $B_{p q}^{s}(\Gamma, \mu)$ by traces

$$
B_{p q}^{s}(\Gamma, \mu)=\operatorname{tr}_{\mu} B_{p q}^{s+\frac{2-d}{p}}\left(\mathbb{T}^{2}\right), \quad 1<p<\infty, 0<q<\infty, 0<s<\infty .
$$

We prefer the periodic setting since we are interested to extend the theory to a closed snowflake. The second way is to use the snowflaked transform $H: \mathbb{T} \rightarrow \Gamma, \mathbb{T}-1$-torus and define $\mathbb{B}_{p q}^{s}(\Gamma)$ by

$$
\mathbb{B}_{p q}^{s}(\Gamma)=\left\{f \circ H^{-1}: f \in B_{p q}^{s}(\mathbb{T})\right\}=B_{p q}^{s}(\mathbb{T}) \circ H^{-1}
$$

where $B_{p q}^{s}(\mathbb{T})$ are periodic Besov spaces. 
The question arises how the function spaces $B_{p q}^{s}(\Gamma, \mu)$ and $\mathbb{B}_{p q}^{s}(\Gamma)$ are interrelated. We concentrate mainly on the case $1<p=q<\infty, 0<s<1$. In particular, we shift the characterization in terms of Daubechies wavelets from $\left(\mathbb{T}, \rho=|x-y|^{1 / d}, \mu_{L}\right)$, [7, p. 360], to $\Gamma$.

This paper is organized as follows. In Section 2, we describe the trace method of defining Besov spaces. In Section 3, we present the wavelet characterization of the periodic Besov spaces $B_{p q}^{s}(\mathbb{T})$ and then shift it to $\Gamma$. In Section 4 , we compare $B_{p p}^{s}(\Gamma, \mu)$ and $\mathbb{B}_{p p}^{s}(\Gamma)$. The main result is contained in Theorem 3.

\section{Trace spaces}

\subsection{Periodic Besov spaces on $\mathbb{T}^{n}$. Let}

$$
\mathbb{T}^{n}=\left\{x=\left(x_{1}, \ldots, x_{n}\right) \in \mathbb{R}^{n}: 0 \leq x_{i} \leq 1, i=1, \ldots, n\right\}
$$

$x \in \mathbb{T}^{n}$ and $y \in \mathbb{T}^{n}$ are identified if and only if $x-y=k, k=\left(k_{1}, \ldots, k_{n}\right) \in$ $\mathbb{Z}^{n}$.

By $D\left(\mathbb{T}^{n}\right)$, we denote the collection of all complex-valued infinitely differentiable functions on $\mathbb{T}^{n}$. The topology in $D\left(\mathbb{T}^{n}\right)$ is generated by the family of semi-norms $\sup _{x \in \mathbb{T}^{n}}\left|D^{\alpha} f(x)\right|$, where $\alpha$ is an arbitrary multi-index. $D^{\prime}\left(\mathbb{T}^{n}\right)$ is defined to be the topological dual of $D\left(\mathbb{T}^{n}\right)$. Any $f \in D^{\prime}\left(\mathbb{T}^{n}\right)$ can be represented as

$$
f=\sum_{k \in \mathbb{Z}^{n}} a_{k} e^{2 \pi i k x}, x \in \mathbb{T}^{n}, \quad\left(\text { convergence in } D^{\prime}\left(\mathbb{T}^{n}\right)\right)
$$

where the Fourier coefficients $\left\{a_{k}\right\} \subset \mathbb{C}$ are of at most polynomial growth,

$$
\left|a_{k}\right| \leq c(1+|k|)^{\kappa}, \text { for some } c>0, \kappa>0 \text { and all } k \in \mathbb{Z}^{n} .
$$

Definition 1. Let $\varphi=\left\{\varphi_{j}\right\}_{j=0}^{\infty}$ be a dyadic resolution of unity, $s \in \mathbb{R}$, $0<p \leq \infty, 0<q \leq \infty$ and

$$
\left\|f \mid B_{p q}^{s}\left(\mathbb{T}^{n}\right)\right\|=\left(\sum_{j=0}^{\infty} 2^{j s q}\left\|\sum_{k \in \mathbb{Z}^{n}} \varphi_{j}(k) a_{k} e^{2 \pi i k x} \mid L_{p}\left(\mathbb{T}^{n}\right)\right\|^{q}\right)^{\frac{1}{q}}
$$

(with the usual modification if $q=\infty$ ). Then the Besov space $B_{p q}^{s}\left(\mathbb{T}^{n}\right)$ consists of all $f \in D^{\prime}\left(\mathbb{T}^{n}\right)$ such that $\left\|f \mid B_{p q}^{s}\left(\mathbb{T}^{n}\right)\right\|<\infty,[6$, Chapter 3]. 


\subsection{Trace spaces.}

Definition 2. A compact set $\Gamma$ in $\mathbb{R}^{n}$ is called a $d$-set with $0<d \leq n$ if there is a Radon measure $\mu$ in $\mathbb{R}^{n}$ with support $\Gamma$ such that for some positive constants $c_{1}$ and $c_{2}$

$$
c_{1} r^{d} \leq \mu(B(\gamma, r)) \leq c_{2} r^{d}, \quad \gamma \in \Gamma, 0<r<1,0<d \leq n
$$

where $B(x, r)$ is a ball in $\mathbb{R}^{n}$ centred at $x \in \mathbb{R}^{n}$ and of radius $r>0$.

If $\Gamma$ is a $d$-set, then the restriction to $\Gamma$ of the $d$-dimensional Hausdorff measure satisfies (1) and any measure $\mu$ satisfying (1) is equivalent to $\left.\mathrm{H}^{d}\right|_{\Gamma}$.

When $\Gamma$ is the Koch curve, it is a $d$-set in $\mathbb{R}^{2}$ with $d=\frac{\log 4}{\log 3}$. Moreover $\Gamma$ is a subset of $\mathbb{T}^{2}$. In order to avoid problems in the endpoints $(0,0)$ and $(1,0)$ of $\Gamma$, we define the Besov spaces as a trace of the periodic Besov spaces $B_{p q}^{s}\left(\mathbb{T}^{2}\right)$.

Suppose that for some

$$
s>0, \quad 1<p<\infty, \quad 0<q<\infty
$$

there is a constant $c>0$ such that

$$
\int_{\Gamma}|\varphi(\gamma)| \mu(d \gamma) \leq c\left\|\varphi \mid B_{p q}^{s}\left(\mathbb{T}^{2}\right)\right\|, \text { for all } \varphi \in D\left(\mathbb{T}^{2}\right)
$$

where $\varphi(\gamma)$ denotes the pointwise trace of $\varphi \in D\left(\mathbb{T}^{2}\right)$ on $\Gamma$ (sometimes we wright $\left.\varphi\right|_{\Gamma}$ to denote the pointwise trace of $\left.\varphi\right) . \quad D\left(\mathbb{T}^{2}\right)$ is dense in $B_{p q}^{s}\left(\mathbb{T}^{2}\right)$. Then (3) can be extended by completion to any $f \in B_{p q}^{s}\left(\mathbb{T}^{2}\right)$ and the resulting function on $\Gamma$ is denoted by $\operatorname{tr}_{\mu} f$. By standard arguments, it is independent of the approximation of $f$ in $B_{p q}^{s}\left(\mathbb{T}^{2}\right)$ by $D\left(\mathbb{T}^{2}\right)$-functions. Any function $g \in \operatorname{tr}_{\mu} B_{p q}^{s}\left(\mathbb{T}^{2}\right) \subset L_{1}(\Gamma, \mu)$ is quasi-normed by

$$
\left\|g \mid \operatorname{tr}_{\mu} B_{p q}^{s}\left(\mathbb{T}^{2}\right)\right\|=\inf \left\{\left\|f \mid B_{p q}^{s}\left(\mathbb{T}^{2}\right)\right\|: \operatorname{tr}_{\mu} f=g\right\}
$$

If one has (3) for some $s, p, q$ satisfying (2), then one has also (3) for all spaces $B_{p v}^{s+\varepsilon}\left(\mathbb{T}^{n}\right)$ with $\varepsilon>0$ and $0<v \leq \infty$.

From the Corollary 1.175 in [7], it follows that the trace operator

$$
\operatorname{tr}_{\mu}: B_{p q}^{s}\left(\mathbb{T}^{2}\right) \hookrightarrow L_{1}(\Gamma, \mu)
$$

exists if $s>\frac{2-d}{p}$. This justifies the following definition 
Definition 3. Let $\Gamma$ be the Koch curve. Let $1<p<\infty, 0<q<\infty$ and $s>0$. Then

$$
B_{p q}^{s}(\Gamma, \mu)=\operatorname{tr}_{\mu} B_{p q}^{s+\frac{2-d}{p}}\left(\mathbb{T}^{2}\right) .
$$

Here $B_{p q}^{s}(\Gamma, \mu)$ are considered as subsets of $L_{1}(\Gamma, \mu)$.

Let

$$
B_{p}^{s}(\Gamma)=B_{p p}^{s}(\Gamma, \mu), \quad 1<p<\infty, \quad 0<s<1 .
$$

It was shown in [3] that $B_{p}^{s}(\Gamma)$ with $1<p<\infty$ and $0<s<1$ can be equivalently normed by $\left\|f \mid B_{p}^{s}(\Gamma)\right\|_{*}$ with

$$
\left\|\left.f\left|B_{p}^{s}(\Gamma) \|_{*}^{p}=\int_{\Gamma}\right| f(\gamma)\right|^{p} \mu(d \gamma)+\int_{\Gamma \times \Gamma} \frac{|f(\gamma)-f(\delta)|^{p}}{|\gamma-\delta|^{d+s p}} \mu(d \gamma) \mu(d \delta)\right.
$$

with $\mu=\left.\mathrm{H}^{d}\right|_{\Gamma}$.

\section{Wavelets on $\mathbb{T}$ and $\Gamma$}

3.1. Self-similar sets and the snowflaked transform. Let $K$ be a self-similar set in $\mathbb{R}^{n}$ with respect to the contractions $\left\{F_{i}\right\}_{i=1}^{N}$, $K=\bigcup_{i=1}^{N} F_{i}(K)$. We can use iterations of the maps $F_{i}$ to give the "address" of a point in $K$. We introduce the following spaces: let $\Sigma$ be a set of all infinite sequences

$$
\Sigma=\left\{\left(\omega_{1}, \omega_{2}, \ldots\right): \omega_{i} \in\{1,2, \ldots, N\}\right\}
$$

We use $W_{m}$ to denote the collection of words of length $m$ :

$$
W_{m}=\left\{\left(w_{1}, w_{2}, \ldots, w_{m}\right): w_{i} \in\{1,2, \ldots, N\}\right\} .
$$

Set $W_{*}=\bigcup_{m=0}^{\infty} W_{m}$. We write $K_{w}=F_{w}(K)$ for $F_{w_{1}} \circ F_{w_{2}} \circ \ldots \circ F_{w_{m}}(K)$, where $w=\left(w_{1}, w_{2}, \ldots, w_{m}\right) \in W_{*}$. For any $\omega=\left(\omega_{1}, \omega_{2}, \ldots\right) \in \Sigma$ define $\pi: \Sigma \rightarrow K$ by

$$
\pi(\omega)=\bigcap_{m=1}^{\infty} K_{\omega_{1} \omega_{2} \ldots \omega_{m}}
$$

see $[4$, Ch. 1.2]. 
The unit interval $\mathrm{I}=[0,1]$ can be considered as a self-similar set with respect to the similarities $T_{i}: \mathbb{R} \rightarrow \mathbb{R}, i=1,2$,

$$
T_{1}(x)=\frac{1}{2} x, \quad T_{2}(x)=\frac{1}{2} x+\frac{1}{2} .
$$

The Koch curve $\Gamma$ is a self-similar set with respect to the similarities $F_{i}: \mathbb{R}^{2} \rightarrow \mathbb{R}^{2}, i=1,2$

$$
\begin{aligned}
& F_{1}\left(\begin{array}{l}
x \\
y
\end{array}\right)=\left(\begin{array}{cc}
\frac{1}{2} & \frac{1}{2 \sqrt{3}} \\
\frac{1}{2 \sqrt{3}} & -\frac{1}{2}
\end{array}\right)\left(\begin{array}{l}
x \\
y
\end{array}\right), \\
& F_{2}\left(\begin{array}{l}
x \\
y
\end{array}\right)=\left(\begin{array}{cc}
\frac{1}{2} & -\frac{1}{2 \sqrt{3}} \\
-\frac{1}{2 \sqrt{3}} & -\frac{1}{2}
\end{array}\right)\left(\begin{array}{l}
x \\
y
\end{array}\right)+\left(\begin{array}{c}
\frac{1}{2} \\
\frac{1}{2 \sqrt{3}}
\end{array}\right) .
\end{aligned}
$$

We denote a mapping $\pi$ corresponding to I by $\pi_{\mathrm{I}}$ and to $\Gamma$ by $\pi_{\Gamma}$,

$$
\pi_{\mathrm{I}}(\omega)=\bigcap_{m=1}^{\infty} \mathrm{I}_{\omega_{1} \omega_{2} \ldots \omega_{m}}, \quad \pi_{\Gamma}(\omega)=\bigcap_{m=1}^{\infty} \Gamma_{\omega_{1} \omega_{2} \ldots \omega_{m}} .
$$

The mapping

$$
H=\pi_{\Gamma} \circ \pi_{\mathrm{I}}^{-1}
$$

is a homeomorphism between I and $\Gamma$. It is called the snowflaked transform. Note that

$$
|H(x)-H(y)| \sim|x-y|^{\frac{1}{d}},
$$

[7, Proposition 8.16].

Since the 1 -torus $\mathbb{T}$ can be identified in the usual way with the unit interval, it can be regarded as a self-similar set with respect to $T_{1}$ and $T_{2}$.

3.2. Self-similar measures. Let $p_{1}, p_{2}, \ldots p_{N}$ be numbers such that

$$
\sum_{i=1}^{N} p_{i}=1, \quad 0<p_{i}<1
$$

Then we can define the probability measure $\mu$ with the weight $\left(p_{1}, p_{2}, \ldots, p_{N}\right)$ on the hierarchy of sets by repeated subdivision of the measure in the ratio $p_{1}: p_{2}: \ldots: p_{N}$, so that

$$
\mu\left(K_{w_{1} w_{2} \ldots w_{m}}\right)=p_{w_{1}} p_{w_{2}} \ldots p_{w_{m}}
$$

and this extends to a Borel measure supported by $K$, [2, Ch. 2.2]. 
Figure 1. The snowflaked transform
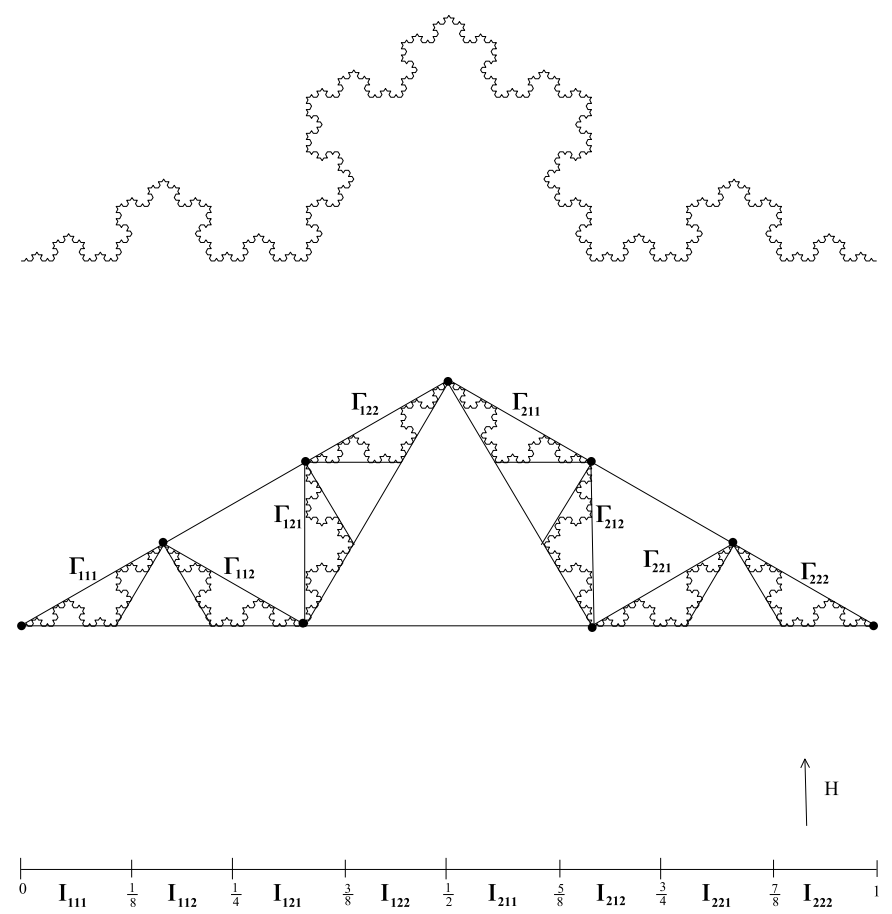

If $\left\{F_{i}\right\}_{i=1}^{N}$ are similarities with factors $r_{i}, i=1,2, \ldots, N$ and $s$ is the unique number with $\sum_{i=1}^{N} r_{i}^{s}=1$, then $\mu$ with weight $\left(r_{1}^{s}, r_{2}^{s}, \ldots, r_{N}^{s}\right)$ is the measure equivalent to the restriction $\left.\mathrm{H}^{s}\right|_{K}$ of the Hausdorff measure $\mathrm{H}^{s}$ in $\mathbb{R}^{n}$ to $K$, [4, Theorem 1.5.7]. When $K$ is the Koch curve $\Gamma$, then the measure $\mu$ with the weight $\left(\frac{1}{2}, \frac{1}{2}\right)$ is equivalent to $\left.\mathrm{H}^{\frac{\ln 4}{\ln 3}}\right|_{\Gamma}$. When $K$ is the unit interval I, measure $\nu$ with the weight $\left(\frac{1}{2}, \frac{1}{2}\right)$ is the Lebesgue measure.

Since the image of the measure $\nu$ under a mapping $H$ is the measure $\mu$, one has for a function $\tilde{f}$ defined on $\Gamma$

$$
\int_{\Gamma} \tilde{f}(\gamma) \mu(d \gamma)=\int_{0}^{1}(\tilde{f} \circ H)(x) \nu(d x)=\int_{0}^{1}(\tilde{f} \circ H)(x) d x,
$$


[5, Theorem 1.19].

\subsection{Wavelet characterization of $B_{p}^{s}(\mathbb{T})$ and $\mathbb{B}_{p}^{s}(\Gamma)$. Let}

$$
\mathbb{B}_{p q}^{s}(\Gamma)=\left\{f \circ H^{-1}: f \in B_{p q}^{s}(\mathbb{T})\right\}=B_{p q}^{s}(\mathbb{T}) \circ H^{-1}
$$

By $B_{p}^{s}(\mathbb{T})$ and $\mathbb{B}_{p}^{s}(\Gamma), 1<p<\infty, 0<s<1$ we denote the spaces $B_{p p}^{s}(\mathbb{T})$ and $\mathbb{B}_{p p}^{s}(\Gamma)$ respectively. We are interested in wavelet expansions for the spaces $\mathbb{B}_{p}^{s}(\Gamma)$. We start with the wavelet characterization of $B_{p}^{s}(\mathbb{T})$ and then transfer it with the help of mapping $H$ to $\Gamma$.

Let $C^{u}(\mathbb{R}), u \in \mathbb{N}$ denote the collection of all complex-valued continuous functions on $\mathbb{R}$ having continuous bounded derivatives up to order $u$ inclusively. Let $\psi_{F} \in C^{u}(\mathbb{R})$ and $\psi_{M} \in C^{u}(\mathbb{R})$ be a father and a mother Daubechies wavelet on $\mathbb{R}$ respectively. Define $\psi_{F}^{k}$ and $\psi_{j}^{k}$ by

$$
\psi_{F}^{k}=\psi_{F}(x-k), \quad \psi_{j}^{k}(x)=2^{\frac{j}{2}} \psi_{M}\left(2^{j} x-k\right), \quad j \in \mathbb{N}_{0}, k \in \mathbb{Z} .
$$

Then $\left\{\psi_{F}^{k}, \psi_{j}^{k}\right\}_{j \in \mathbb{N}_{0}, k \in \mathbb{Z}}$ is an orthonormal system in $L_{2}(\mathbb{R})$. Let $L \in \mathbb{N}$. One can replace $\psi_{F}$ and $\psi_{M}$ by

$$
\psi_{F}^{L}(\cdot)=\psi_{F}\left(2^{L} \cdot\right), \quad \psi_{M}^{L}(\cdot)=\psi_{M}\left(2^{L} \cdot\right)
$$

$\psi_{F}^{k}$ and $\psi_{j}^{k}$ by

$$
\psi_{F}^{L, k}(x)=2^{\frac{L}{2}} \psi_{F}\left(2^{L} x-k\right), \quad \psi_{j}^{L, k}(x)=2^{\frac{j+L}{2}} \psi_{M}\left(2^{j+L} x-k\right), k \in \mathbb{Z} .
$$

We choose and fix $L$ such that

$$
\operatorname{supp} \psi_{F}^{L} \subset\left\{x:|x|<\frac{1}{2}\right\}, \operatorname{supp} \psi_{M}^{L} \subset\left\{x:|x|<\frac{1}{2}\right\} .
$$

Then one has

$$
\operatorname{supp} \psi_{j}^{L, 0}=\operatorname{supp} \psi_{M}^{L}\left(2^{j} \cdot\right) \subset\left\{x:|x|<\frac{1}{2^{j+1}}\right\} .
$$

Let

$$
\mathbb{P}_{j}=\left\{m \in \mathbb{Z}: 0 \leq m<2^{j+L}\right\}, \quad j \in \mathbb{N}_{0}
$$

Given the functions $\psi_{F}^{L, k}, \psi_{j}^{L, k}$ on the real line we can construct their 1 -periodic counterparts by the procedure

$$
\psi_{F, p e r}^{L, k}(x)=\sum_{l=-\infty}^{\infty} \psi_{F}^{L, k}(x+l), \quad \psi_{j, p e r}^{L, k}(x)=\sum_{l=-\infty}^{\infty} \psi_{j}^{L, k}(x+l) .
$$


Define $\psi_{F}^{L, k, p e r}$ and $\psi_{j}^{L, k, p e r}$ on the 1 -torus $\mathbb{T}$ by

$$
\psi_{F}^{L, k, p e r}(x)=\psi_{F, p e r}^{L, k}(x), \quad \psi_{j}^{L, k, p e r}(x)=\psi_{j, p e r}^{L, k}(x), \quad x \in \mathbb{T} .
$$

Then according to the Proposition 1.34 in [8]

$$
\left\{\psi_{F}^{L, k^{\prime}, \text { per }}, \psi_{j}^{k, L, \text { per }}, k^{\prime} \in \mathbb{P}_{0}, j \in \mathbb{N}_{0}, k \in \mathbb{P}_{j}\right\}
$$

is an orthornomal basis in $L_{2}(\mathbb{T})$.

It is easy to see that

$$
\psi_{j}^{L, k, p e r}(x)=\psi_{j}^{L, 0, p e r}\left(x-\frac{k}{2^{j+L}}\right), k \in \mathbb{P}_{j},
$$

on $\mathbb{T}$ with the usual interpretation.

The shift operation is well-defined on the real line, but it can not be defined on the Koch curve. Therefore we would like to replace this operation in order to be able to construct its counterpart on the Koch curve. First of all when $j$ is fixed, the 1-torus $\mathbb{T}$ treated as a self-similar set can be represented as follows

$$
\mathbb{T}=\bigcup_{w \in W_{j+L}} \mathbb{T}_{w}
$$

Let us introduce the order relation on the set $W_{j+L}$ of words of length $j+L$. We say that $v=\left(v_{1}, \ldots, v_{j+L}\right)$ is less than $w=\left(w_{1}, \ldots, w_{j+L}\right)$ if and only if the first $v_{i}$ which is different from $w_{i}$ is less than $w_{i}$ :

$$
v<w \Leftrightarrow v_{\min \left\{i: w_{i} \neq v_{i}\right\}}<w_{\min \left\{i: w_{i} \neq v_{i}\right\}} .
$$

The words are ordered in such a way that whenever $u$ follows $w$ in $W_{j+L}$, the interval $\mathbb{T}_{u}$ is the right neighbour of $\mathbb{T}_{w}$. We agree that $\mathbb{T}_{j+L}^{11 \ldots 1}$ is the right neighbour of $\mathbb{T}^{22 \ldots 2}$.

Then we notice that

$$
x-\frac{k}{2^{j+L}}=2^{-(j+L)}\left(2^{j+L} x-k\right) .
$$

When $k$ is fixed, there is a unique sequence of contractions

$$
T_{w_{1}}, T_{w_{2}}, \ldots, T_{w_{j+L}}, \quad w=\left(w_{1}, w_{2}, \ldots, w_{j+L}\right) \in W_{j+L}
$$

such that

$$
2^{-(j+L)}\left(2^{j+L} x-k\right)=T_{1}^{j+L} \circ T_{w_{j+L}}^{-1} \circ \ldots \circ T_{w_{2}}^{-1} \circ T_{w_{1}}^{-1}(x) .
$$




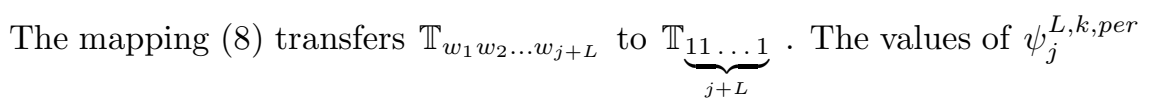
on $\mathbb{T}_{u}$, where $u=\left(u_{1}, u_{2}, \ldots u_{j+L}\right)$ follows $w$, coincide with the values of $\psi_{j}^{L, 0, p e r}$ on $\mathbb{T}_{\underbrace{11 \ldots 12}_{j+L-1}}$. Thus there is the following connection between functions $\psi_{j}^{L, k, p e r}$ and $\psi_{j}^{L, 0, p e r}$ :

$$
\begin{aligned}
& \psi_{j}^{L, k, p e r}(x)= \\
& = \begin{cases}\psi_{j}^{L, 0, \text { per }} \circ T_{1}^{j+L} \circ T_{w_{j+L}}^{-1} \circ \ldots \circ T_{w_{2}}^{-1} \circ T_{w_{1}}^{-1}(x), & \text { if } x \in \mathbb{T}_{w_{1} w_{2} \ldots w_{j+L}}, \\
\psi_{j}^{L, 0, p e r} \circ T_{1}^{j+L-1} \circ T_{2} \circ T_{u_{j+L}}^{-1} \circ \ldots \circ T_{u_{2}}^{-1} \circ T_{u_{1}}^{-1}(x), & \text { if } x \in \mathbb{T}_{u_{1} u_{2} \ldots u_{j+L}}, \\
\ldots & \end{cases}
\end{aligned}
$$

where ... indicates the procedure of assigning to $\psi_{j}^{L, k, p e r}$ on each next right neighbour of $\mathbb{T}_{u}$ the values of $\psi_{j}^{L, 0, p e r}$ on each next right neighbour of $\mathbb{T}_{\underbrace{11 \ldots 1}_{j+L-1}}$.

Let us simplify the notation and denote the functions $\psi_{F}^{L, k^{\prime}, p e r}$ and $\psi_{j}^{L, k, p e r}$ by

$$
\begin{aligned}
\psi_{F, w} & =\psi_{F, w_{1} w_{2} \ldots w_{L}}, \quad w \in W_{L}, \\
\psi_{w} & =\psi_{w_{1} w_{2} \ldots w_{j+L}}, \quad j \in \mathbb{N}_{0}, w \in W_{j+L}
\end{aligned}
$$

respectively, where $w$ is chosen according to (8). Now we transfer the functions $\psi_{F, w}, w \in W_{L}, \psi_{w}, w \in \bigcup_{j \in \mathbb{N}_{0}} W_{j+L}$ from $\mathbb{T}$ to the Koch curve $\Gamma$. Define $\widetilde{\psi}_{F, w}$ and $\widetilde{\psi}_{w}$ by

$$
\begin{aligned}
\widetilde{\psi}_{F, w}(\gamma) & =\psi_{F, w} \circ H^{-1}(\gamma) \\
\widetilde{\psi}_{w}(\gamma) & =\psi_{w} \circ H^{-1}(\gamma)
\end{aligned}
$$

From (7) follows that the system $\left\{\widetilde{\psi}_{F, v}, \widetilde{\psi}_{w}\right\}_{v \in W_{L}, w \in \bigcup_{j \in \mathbb{N}_{0}} W_{j+L}}$ is orthonormal in $L_{2}(\Gamma, \mu)$.

Let $\gamma \in \Gamma_{w_{1} w_{2} \ldots w_{j+L}}$. Then there is an $\omega \in \Sigma$ such that

$$
\gamma=\bigcap_{m=1}^{\infty} \Gamma_{w_{1} w_{2} \ldots w_{j+L} \omega_{1} \omega_{2} \ldots \omega_{m}}
$$


Recall that $\gamma \in \Gamma$ corresponds to $x=H^{-1}(\gamma) \in \mathbb{T}_{w_{1} w_{2} \ldots w_{j+L}}$ given by

$$
x=\bigcap_{m=1}^{\infty} \mathbb{T}_{w_{1} w_{2} \ldots w_{j+L} \omega_{1} \omega_{2} \ldots \omega_{m}} .
$$

The connection between the values of $\widetilde{\psi}_{w_{1} w_{2} \ldots w_{j+L}}$ and $\widetilde{\psi}_{\underbrace{11 \ldots 1}_{j+L}}$ on $\Gamma_{w_{1} w_{2} \ldots w_{j+L}}$ is the following:

$$
\begin{aligned}
& \widetilde{\psi}_{w_{1} w_{2} \ldots w_{j+L}}(\gamma)=\psi_{w_{1} w_{2} \ldots w_{j+L}} \circ H^{-1}(\gamma)= \\
& =\psi_{j+L} \underbrace{}_{11 \ldots 1} \circ T_{1}^{j+L} \circ T_{w_{j+L}}^{-1} \circ \ldots \circ T_{w_{2}}^{-1} \circ T_{w_{1}}^{-1}\left(\bigcap_{m=1}^{\infty} \mathbb{T}_{w_{1} w_{2} \ldots w_{j+L} \omega_{1} \omega_{2} \ldots \omega_{m}}\right)= \\
& =\psi_{j+L}^{11 \ldots 1}\left(\bigcap_{m=1}^{\infty} \mathbb{T}_{\underbrace{11 \ldots 1}_{j+L}} \omega_{1} \omega_{2} \ldots \omega_{m}\right)=\psi_{\underbrace{11 \ldots 1}_{j+L}} \circ H^{-1}\left(\bigcap_{m=1}^{\infty} \Gamma_{j+L}^{11 \ldots 1} \omega_{1} \omega_{2} \ldots \omega_{m}\right)= \\
& =\widetilde{\psi}_{\underbrace{11 \ldots 1}_{j+L}}\left(\bigcap_{m=1}^{\infty} \Gamma_{\underbrace{11 \ldots 1}_{j+L}} \omega_{1} \omega_{2} \ldots \omega_{m}\right)= \\
& =\widetilde{\psi}_{\underbrace{1 \ldots 1}_{j+L}} \circ F_{1}^{j+L} \circ F_{w_{j+L}}^{-1} \circ \ldots \circ F_{w_{2}}^{-1} \circ F_{w_{1}}^{-1}\left(\bigcap_{m=1}^{\infty} \Gamma_{w_{1} w_{2} \ldots w_{j+L} \omega_{1} \omega_{2} \ldots \omega_{m}}\right)= \\
& =\widetilde{\psi}_{\underbrace{1 \ldots 1}_{j+L}} \circ F_{1}^{j+L} \circ F_{w_{j+L}}^{-1} \circ \ldots \circ F_{w_{2}}^{-1} \circ F_{w_{1}}^{-1}(\gamma) \text {. }
\end{aligned}
$$

In the same way we can follow the connection between the values of $\widetilde{\psi}_{w_{1} w_{2} \ldots w_{j+L}}$ and $\widetilde{\psi}_{\underbrace{11 \ldots 1}_{j+L}}$ on other $\Gamma_{v}, v \in W_{j+L}$. So we get

$$
\begin{aligned}
& \widetilde{\psi}_{w}(\gamma)= \\
& = \begin{cases}\widetilde{\psi}_{\underbrace{11 \ldots 1}_{j+L}} \circ F_{1}^{j+L} \circ F_{w_{j+L}}^{-1} \circ \ldots \circ F_{w_{2}}^{-1} \circ F_{w_{1}}^{-1}(\gamma), & \text { if } \gamma \in \Gamma_{w_{1} w_{2} \ldots w_{j+L}}, \\
\widetilde{\psi}_{\underbrace{11 \ldots 1}_{j+L}}^{\widetilde{p}_{1}} \circ F_{1}^{j+L-1} \circ F_{2} \circ F_{u_{j+L}}^{-1} \circ \ldots \circ F_{u_{2}}^{-1} \circ F_{u_{1}}^{-1}(\gamma), & \text { if } \gamma \in \Gamma_{u_{1} u_{2} \ldots u_{j+L}}, \\
\ldots & \end{cases}
\end{aligned}
$$

Similarly for $\widetilde{\psi}_{F, w}$.

According to $[8$, Ch. 1.3.3 $]$ the following theorem holds. 
Theorem 1. Let $1<p<\infty, 0<s<1$ and $u>s$. Let $f \in L_{p}(\mathbb{T})$. Then $f \in B_{p}^{s}(\mathbb{T})$ if, and only if, it can be represented as

$$
f=\sum_{w \in W_{L}} a_{w} 2^{-\frac{L}{2}} \psi_{F, w}+\sum_{j=0}^{\infty} \sum_{w \in W_{j+L}} b_{w} 2^{-\frac{j+L}{2}} \psi_{w},
$$

unconditional convergence being in $L_{p}(\mathbb{T})$. Furthermore this representation is unique,

$$
\begin{aligned}
& a_{w}=2^{\frac{L}{2}}\left(f, \psi_{F, w}\right)_{\mathbb{T}}, \quad w \in W_{L}, \\
& b_{w}=2^{\frac{j+L}{2}}\left(f, \psi_{w}\right)_{\mathbb{T}}, \quad w \in W_{j+L}
\end{aligned}
$$

and

$$
I: f \rightarrow\left\{a_{w}, w \in W_{L}, b_{w}, w \in W_{j+L}, j \in \mathbb{N}_{0}\right\}
$$

is an isomorphic map of $B_{p}^{s}(\mathbb{T})$ onto the sequence space such that

$$
\sum_{w \in W_{L}}\left|a_{w}\right|^{p}+\sum_{j=0}^{\infty} 2^{j\left(s-\frac{1}{p}\right) p} \sum_{w \in W_{j+L}}\left|b_{w}\right|^{p}<\infty .
$$

Similar theorem holds for the spaces $\mathbb{B}_{p}^{s}(\Gamma)$.

Theorem 2. Let $1<p<\infty, 0<s<1$ and $u>s$. Let $\tilde{f} \in L_{p}(\Gamma)$. Then $\tilde{f} \in \mathbb{B}_{p}^{s}(\Gamma)$ if, and only if, it can be represented as

$$
\tilde{f}=\sum_{w \in W_{L}} a_{w} 2^{-\frac{L}{2}} \tilde{\psi}_{F, w}+\sum_{j=0}^{\infty} \sum_{w \in W_{j+L}} b_{w} 2^{-\frac{j+L}{2}} \tilde{\psi}_{w}
$$

unconditional convergence being in $L_{p}(\Gamma)$. Furthermore this representation is unique,

$$
\begin{aligned}
& a_{w}=2^{\frac{L}{2}}\left(\tilde{f}, \tilde{\psi}_{F, w}\right)_{\Gamma}, \quad w \in W_{L}, \\
& b_{w}=2^{\frac{j+L}{2}}\left(\tilde{f}, \tilde{\psi}_{w}\right)_{\Gamma}, \quad w \in W_{j+L}
\end{aligned}
$$

and

$$
I: \tilde{f} \rightarrow\left\{a_{w}, w \in W_{L}, b_{w}, w \in W_{j+L}, j \in \mathbb{N}_{0}\right\}
$$

is an isomorphic map of $\mathbb{B}_{p}^{s}(\Gamma)$ onto the sequence space such that

$$
\sum_{w \in W_{L}}\left|a_{w}\right|^{p}+\sum_{j=0}^{\infty} 2^{j\left(s-\frac{1}{p}\right) p} \sum_{w \in W_{j+L}}\left|b_{w}\right|^{p}<\infty .
$$




\section{Comparison of $B_{p}^{s}(\Gamma)$ and $\mathbb{B}_{p}^{s}(\Gamma)$}

The spaces $B_{p}^{s}(\mathbb{T})$ can be normed by

$$
\left\|\left.f\left|B_{p}^{s}(\mathbb{T}) \|_{*}^{p}=\int_{0}^{1}\right| f(x)\right|^{p} d x+\int_{0}^{1} \int_{0}^{1} \frac{|f(x)-f(y)|^{p}}{|x-y|^{1+s p}} d x d y .\right.
$$

Since (9) is equivalent to

$$
\int_{\Gamma}|\tilde{f}(\gamma)|^{p} \mu(d \gamma)+\int_{\Gamma} \int_{\Gamma} \frac{|\tilde{f}(\gamma)-\tilde{f}(\delta)|^{p}}{|\gamma-\delta|^{d+s p}} \mu(d \gamma) \mu(d \delta),
$$

where $\tilde{f}=f \circ H^{-1}$, we endow the spaces $\mathbb{B}_{p}^{s}(\Gamma)$ with the norm

$$
\left\|\left.\tilde{f}\left|\mathbb{B}_{p}^{s}(\Gamma) \|_{*}^{p}=\int_{\Gamma}\right| \tilde{f}(\gamma)\right|^{p} \mu(d \gamma)+\int_{\Gamma} \int_{\Gamma} \frac{|\tilde{f}(\gamma)-\tilde{f}(\delta)|^{p}}{|\gamma-\delta|^{d+s d p}} \mu(d \gamma) \mu(d \delta) .\right.
$$

Together with (5) this leads to

$$
B_{p}^{s}(\Gamma)=\mathbb{B}_{p}^{\frac{s}{d}}(\Gamma) .
$$

The analogue of Theorem 2 for the spaces $B_{p}^{s}(\Gamma)$ reads as follows

Theorem 3. Let $1<p<\infty, 0<s<1$ and $u>s$. Let $\tilde{f} \in L_{p}(\Gamma)$. Then $\tilde{f} \in B_{p}^{s}(\Gamma)$ if, and only if, it can be represented as

$$
\tilde{f}=\sum_{w \in W_{L}} a_{w} 2^{-\frac{L}{2}} \tilde{\psi}_{F, w}+\sum_{j=0}^{\infty} \sum_{w \in W_{j+L}} b_{w} 2^{-\frac{j+L}{2}} \tilde{\psi}_{w},
$$

unconditional convergence being in $L_{p}(\Gamma)$. Furthermore this representation is unique,

$$
\begin{aligned}
& a_{w}=2^{\frac{L}{2}\left(\tilde{f}, \tilde{\psi}_{F, w}\right)_{\Gamma}, \quad w \in W_{L},} \\
& b_{w}=2^{\frac{j+L}{2}}\left(\tilde{f}, \tilde{\psi}_{w}\right)_{\Gamma}, \quad w \in W_{j+L}
\end{aligned}
$$

and

$$
I: \tilde{f} \rightarrow\left\{a_{w}, w \in W_{L}, b_{w}, w \in W_{j+L}, j \in \mathbb{N}_{0}\right\}
$$

is an isomorphic map of $B_{p}^{s}(\Gamma)$ onto the sequence space such that

$$
\sum_{w \in W_{L}}\left|a_{w}\right|^{p}+\sum_{j=0}^{\infty}(\sqrt{3})^{j\left(s-\frac{d}{p}\right) p} \sum_{w \in W_{j+L}}\left|b_{w}\right|^{p}<\infty .
$$


Proof. This follows from the observation (11) and

$$
2^{j\left(\frac{s}{d}-\frac{1}{p}\right)}=2^{\frac{1}{d} j\left(s-\frac{d}{p}\right)}=(\sqrt{3})^{j\left(s-\frac{d}{p}\right)} .
$$

\section{References}

[1] D. E. Edmunds and H. Triebel, Function Spaces, Entropy Numbers, Differential Operators, Cambridge Univ. Press, 1996.

[2] K. Falconer, Techniques in Fractal Geometry, John Wiley \& Sons, 1997.

[3] A. Jonsson and H. Wallin, Function Spaces on Subsets of $\mathbb{R}^{n}$, Math. Reports, 2, London, Harwood Acad. Publ., 1984.

[4] J. Kigami, Analysis on Fractals, Cambridge University Press, 2003.

[5] P. Mattila, Geometry of Sets and Measures in Euclidean Spaces, Cambridge Univ. Press, 1995.

[6] H.-J. Schmeisser and H. Triebel, Topics in Fourier Analysis and Function Spaces, Wiley, Chichester, 1987.

[7] H. Triebel, Theory of Function Spaces III, Birkhäuser, Basel, 2006.

[8] H. Triebel, Function Spaces and Wavelets on Domains, European Math. Soc. Publishing House, Zürich, 2008.

Mathematical Institute

Friedrich Schiller University Jena

D-07737 Jena, Germany

(E-mail : maryia.kabanava@uni-jena.de)

(Received : February 2009) 


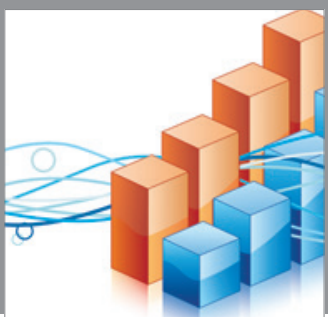

Advances in

Operations Research

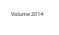

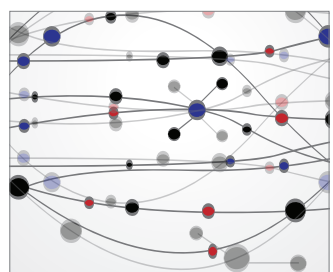

\section{The Scientific} World Journal
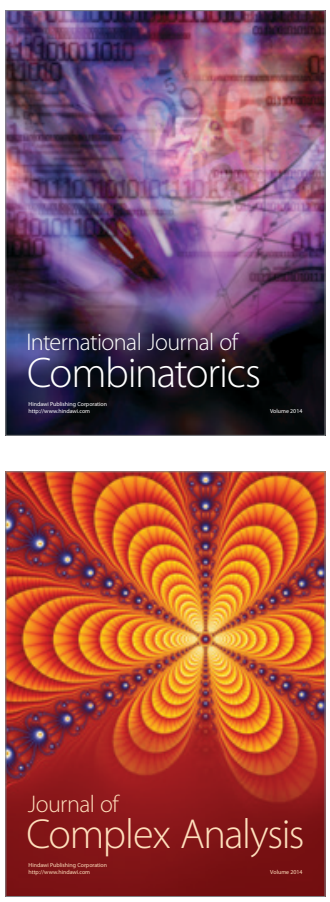

International Journal of

Mathematics and

Mathematical

Sciences
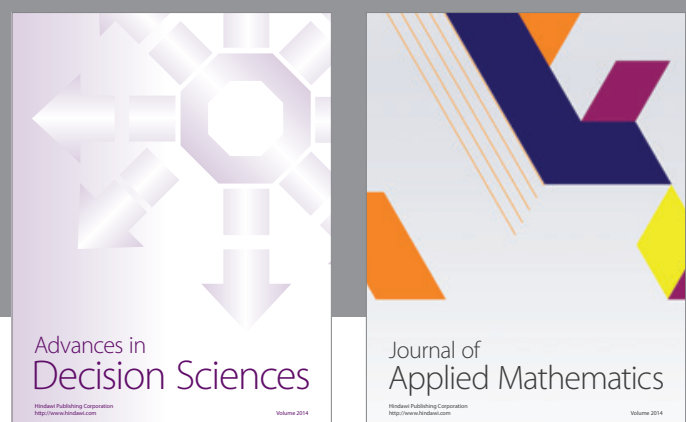

Journal of

Applied Mathematics
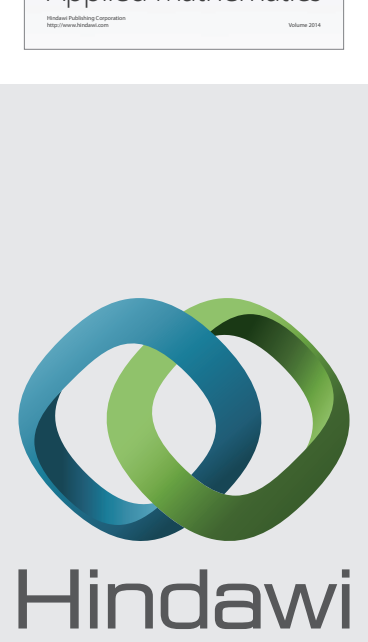

Submit your manuscripts at http://www.hindawi.com
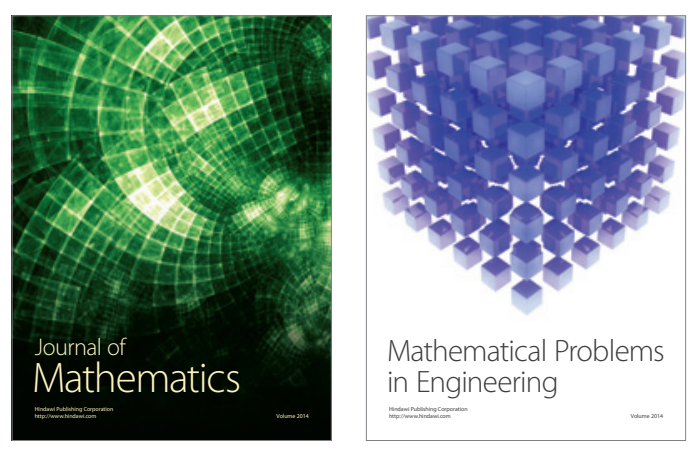

Mathematical Problems in Engineering
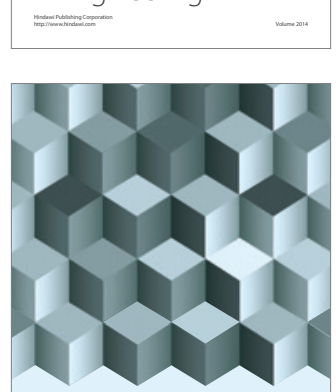

Journal of

Function Spaces
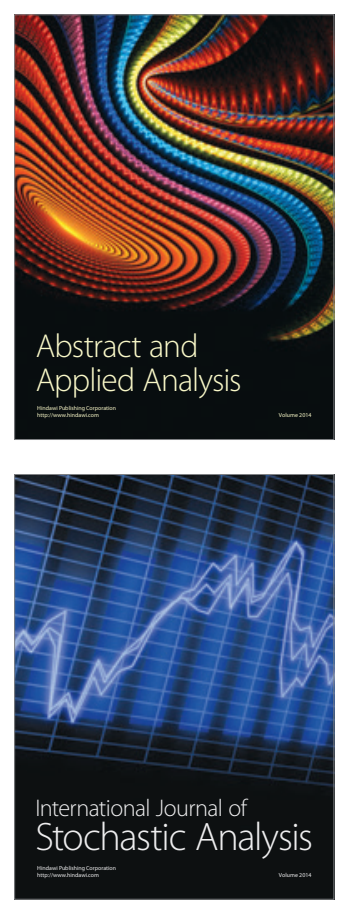

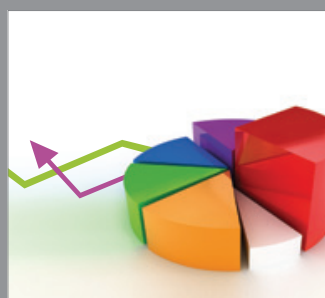

ournal of

Probability and Statistics

Promensencen
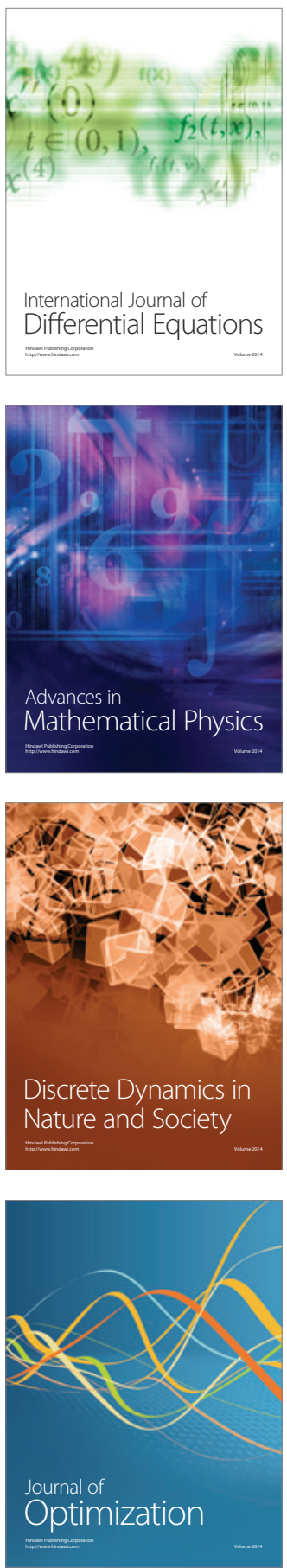